\title{
Diadenosine Tetraphosphate Protects against Injuries Induced by Ischemia and 6-Hydroxydopamine in Rat Brain
}

\author{
Yun Wang, ${ }^{1,2}$ Chen-Fu Chang, ${ }^{1,2}$ Marisela Morales, ${ }^{1}$ Yung-Hsiao Chiang, ${ }^{2}$ Brandon K. Harvey, ${ }^{1}$ Tsung-Ping Su, ${ }^{1}$ \\ Li-I Tsao, ${ }^{1}$ Suyu Chen, ${ }^{1}$ and Christoph Thiemermann ${ }^{3}$ \\ ${ }^{1}$ National Institute on Drug Abuse, National Institutes of Health, Baltimore, Maryland 21224, ${ }^{2}$ Triservice General Hospital, National Defense Medical \\ Center, Taipei, 114 Taiwan, and ${ }^{3}$ William Harvey Research Institute, Queen Mary University, London, EC1 M 6BQ United Kingdom
}

Diadenosine tetraphosphate $\left(\mathrm{AP}_{4} \mathrm{~A}\right)$, an endogenous diadenosine polyphosphate, reduces ischemic injury in the heart. In this study, we report the potent and protective effects of $\mathrm{AP}_{4} \mathrm{~A}$ in rodent models of stroke and Parkinson's disease. $\mathrm{AP}_{4} \mathrm{~A}$, given intracerebroventricularly before middle cerebral artery (MCA) ligation, reduced cerebral infarction size and enhanced locomotor activity in adult rats. The intravenous administration of $\mathrm{AP}_{4} \mathrm{~A}$ also induced protection when given early after MCA ligation. $\mathrm{AP}_{4} \mathrm{~A}$ suppressed terminal deoxynucleotidyl transferase-mediated biotinylated UTP nick end labeling (TUNEL) induced by hypoxia/reperfusion in primary cortical cultures, and reduced both ischemia-induced translocation of mitochondrial cytochrome $c$ and the increase in cytoplasmic caspase-3 activity in vivo. The purinergic $\mathrm{P}_{2} / \mathrm{P}_{4}$ antagonist di-inosine pentaphosphate or $\mathrm{P}_{1}$-receptor antagonist sulfonylphenyl theophylline, but not the $\mathrm{P}_{2}$-receptor antagonist suramin, antagonized the effect of $\mathrm{AP}_{4} \mathrm{~A}$, suggesting that the observed protection is mediated through an antiapoptotic mechanism and the activation of $\mathrm{P}_{1}$ - and $\mathrm{P}_{4}$-purinergic receptors.

$\mathrm{AP}_{4} \mathrm{~A}$ also afforded protection from toxicity induced by unilateral medial forebrain bundle injection of 6-hydroxydopamine (6OHDA). One month after lesioning, vehicle-treated rats exhibited amphetamine-induced rotation. Minimal tyrosine hydroxylase immunoreactivity was detected in the lesioned nigra or striatum. No KCl-induced dopamine release was found in the lesioned striatum. All of these indices of dopaminergic degeneration were attenuated by pretreatment with $\mathrm{AP}_{4} \mathrm{~A}$. In addition, $\mathrm{AP}_{4} \mathrm{~A}$ reduced TUNEL in the lesioned nigra $2 \mathrm{~d}$ after 6-OHDA administration. Collectively, our data suggest that $\mathrm{AP}_{4} \mathrm{~A}$ is protective against neuronal injuries induced by ischemia or 6-OHDA through the inhibition of apoptosis. We propose that $\mathrm{AP}_{4} \mathrm{~A}$ may be a potentially useful target molecule in the therapy of stroke and Parkinson's disease.

Key words: ischemia; stroke; Parkinson's disease; repair; protection; diadenosine tetraphosphate; apoptosis

\section{Introduction}

Diadenosine polyphosphates $\left(\mathrm{AP}_{n} \mathrm{As}\right)$ are a group of compounds that contain two adenosine moieties bridged by three to six phosphates. Diadenosine tetraphosphate $\left(\mathrm{AP}_{4} \mathrm{~A}\right)$, a major representative of $\mathrm{AP}_{n} \mathrm{As}$, has been found to be highly concentrated in tears (Pintor et al., 2002), and it may be involved in extracellular and intracellular signaling in the brain (Oaknin et al., 2001; Emanuelli et al., 1998; Kisselev et al., 1998). $\mathrm{AP}_{4} \mathrm{~A}$ is stored in secretory granules with ATP and can be released after stimulation in a $\mathrm{Ca}^{2+}$-dependent manner (Pivorun and Nordone, 1996) and during insults (Pintor et al., 1995) or oxidative stress (Bochner et al., 1984). $\mathrm{AP}_{4} \mathrm{~A}$ interacts with purinergic $\mathrm{P}_{1^{-}}$(Klishin et al., 1994) and $\mathrm{P}_{2^{-}}$(Lazarowski et al., 1995) receptors. Recent studies have indicated that high-affinity binding sites for $\mathrm{AP}_{4} \mathrm{~A}$ (Pintor et al., 1993), as differentiated from $\mathrm{P}_{1}$ - or $\mathrm{P}_{2}$-receptors, are found in the olfactory bulb, cerebral cortex, and striatum, as well as several other brain areas (Rodriguez-Pascual et al., 1997). These recep-

\footnotetext{
Received March 5, 2003; revised July 3, 2003; accepted July 7, 2003.

This work was supported by the National Institute on Drug Abuse and the National Science Council (Taiwan). We thank Hui Shen, Jenny Chou, and Huilin Chen for their technical assistance.

Correspondence should be addressed to Dr. Yun Wang, National Institute on Drug Abuse, National Institutes of Health, 5500 Nathan Shock Drive, Baltimore, MD 21224. E-mail: ywang@intra.nida.nih.gov.

Copyright $\odot 2003$ Society for Neuroscience $\quad$ 0270-6474/03/237958-08\$15.00/0
}

tors have been identified as purinergic $\mathrm{P}_{4}$-receptors (RodriguezPascual et al., 1997). In the synaptic terminals of rat midbrain and cerebellum, the interaction of $\mathrm{AP}_{n}$ As and $\mathrm{P}_{4}$ receptors cannot be cross-desensitized by ATP (Pintor and Miras-Portugal, 1995). Taken together, these data suggest that $\mathrm{AP}_{4} \mathrm{~A}$ may use unique signal transduction pathways and may activate physiological responses different from those caused by ATP or adenosine.

Previous studies have indicated that $\mathrm{AP}_{4} \mathrm{~A}$ reduces ischemic injury in the heart (Ahmet et al., 2000; Khattab et al., 1998). The role of $\mathrm{AP}_{4} \mathrm{~A}$ in the $\mathrm{CNS}$ is not clear. $\mathrm{AP}_{4} \mathrm{~A}$ can be released from the striatum after the systemic administration of amphetamine (Pintor et al., 1995). $\epsilon-\mathrm{AP}_{4} \mathrm{~A}$, an $\mathrm{AP}_{4} \mathrm{~A}$ analog, has been found to decrease extracellular glutamate levels in the striatum (Oaknin et al., 2001). We now report that $\mathrm{AP}_{4} \mathrm{~A}$ has protective effects in the cortex and midbrain in defined rat models of stroke and Parkinson's disease, which have predictive value for therapeutic development.

\section{Materials and Methods}

In vitro primary neuronal cultures, hypoxia, and cell death. Cells were isolated at embryonic day 15 from timed-pregnant rats. Cells were plated in Neurobasal media containing $B_{27}$ supplement, $200 \mathrm{~mm}$ L-glutamine, and $25 \mu \mathrm{M}$ L-glutamate for $4 \mathrm{~d}$. Cells were treated with $\mathrm{AP}_{4} \mathrm{~A}$ (SigmaAldrich Chemical, St. Louis, MO) or vehicle and incubated for $18 \mathrm{hr}$ in a 


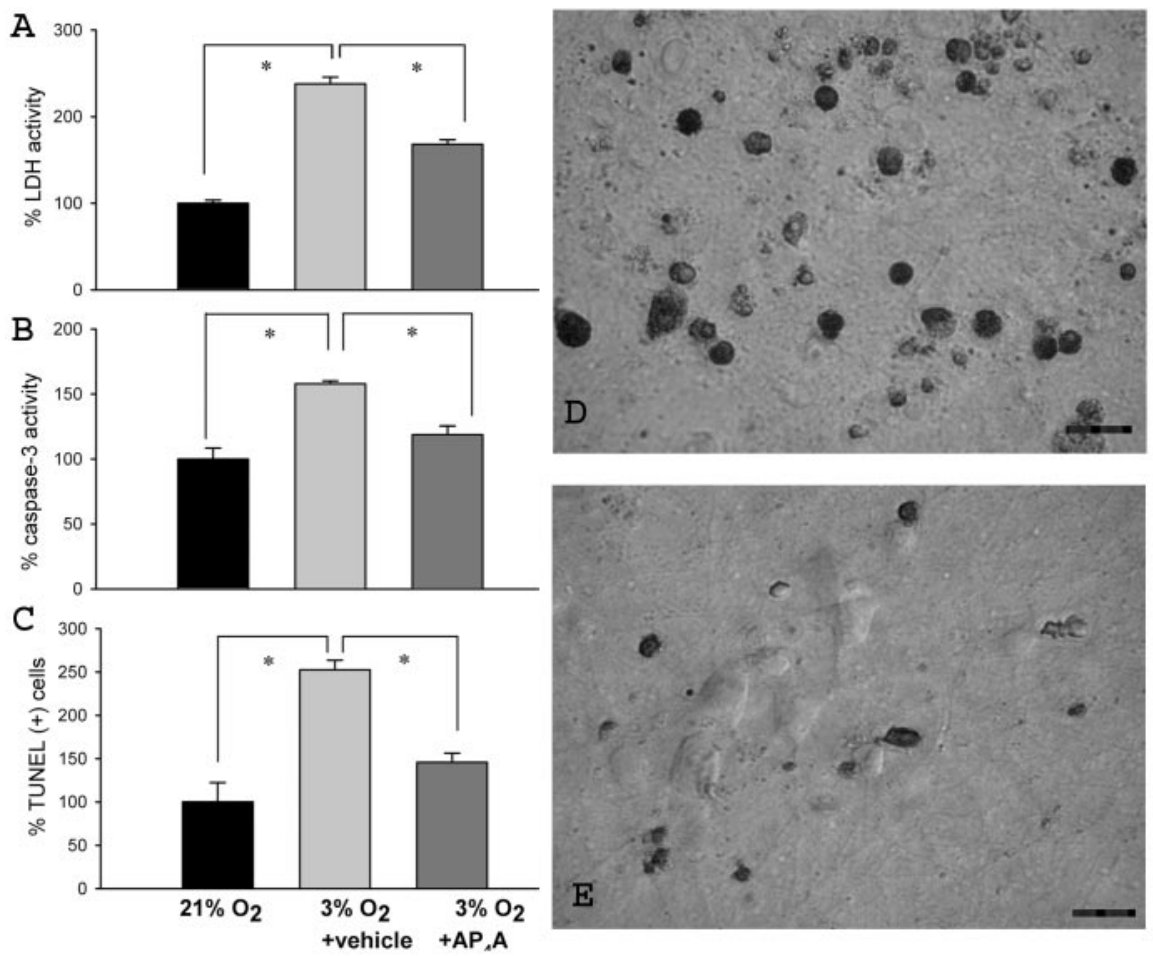

Figure 1. Neuroprotective effects of $A P_{4} A$ against hypoxia/reoxygenation in vitro. Exposure to $A P_{4} A(100 \mathrm{~nm})$ significantly reduced the hypoxia/reoxygenation-induced release of $\operatorname{LDH}(A)$, increase in caspase-3 enzymatic activity $(B)$, and increase in TUNEL labeling in primary cortical cultures $(C){ }^{*} p<0.05$, one-way ANOVA. Photomicrographs demonstrate that hypoxia/ reoxygenation increased TUNEL in primary cortical cells $(D)$ and pretreatment with $\mathrm{AP}_{4} \mathrm{~A}$ reduced the density of TUNEL $(E)$. Scale bars, $20 \mu \mathrm{m}$.

hypoxic incubator $\left(3 \% \mathrm{O}_{2}, 5 \% \mathrm{CO}_{2}\right.$; Thermo Forma). After hypoxia, cells were returned to normoxia conditions $\left(21 \% \mathrm{O}_{2}, 5 \% \mathrm{CO}_{2}\right)$ for $24 \mathrm{hr}$. Media were collected and assayed for lactate dehydrogenase (LDH) activity (Cytotoxicity Detection Kit; Roche Products, Penzberg, Germany). Cultures were assayed for DNA fragmentation using a terminal deoxynucleotidyl transferase-mediated biotinylated UTP nick end labeling (TUNEL)-based method (In Situ Cell Death Detection Kit; Roche) or activated caspase- 3 activity (see below) using the ApoAlert kit (Clontech, Palo, Alto, CA).

Animals and drug administration for in vivo studies. Adult male Sprague Dawley rats were used for this study. For intraventricular administration of $\mathrm{AP}_{4} \mathrm{~A}$, animals were anesthetized with chloral hydrate $\left(0.4 \mathrm{gm} / \mathrm{kg}\right.$, i.p.). $\mathrm{AP}_{4} \mathrm{~A}(3$ or $30 \mathrm{nmol} / 20 \mu \mathrm{l})$ was administered through a Hamilton syringe over $10 \mathrm{~min}$ intracerebroventricularly. The coordinates were $0.8 \mathrm{~mm}$ posterior to the bregma, $1.5 \mathrm{~mm}$ lateral to the midline, and $3.5 \mathrm{~mm}$ below the dura surface. The speed of injection was controlled by a syringe pump at a rate of $2.5 \mu \mathrm{l} / \mathrm{min}$. A systemic injection of $\mathrm{AP}_{4} \mathrm{~A}(10 \mathrm{mg} / \mathrm{kg})$ or vehicle (saline, $\left.1 \mathrm{ml}\right)$ was given through the tail vein at 5-10 min after removal of the ligature on the right MCA (see below).

Brain ischemia/reperfusion. Anesthetized rats were subjected to cerebral ischemia. The ligation of the right middle cerebral artery (MCA) and bilateral common carotids (CCAs) was performed using methods described previously (Chen et al., 1986;Wang et al., 2001a). The right MCA was ligated with 10-0 suture. The ligature was removed after $60 \mathrm{~min}$ of ischemia to allow reperfusion. Body temperature was monitored with a thermistor probe and maintained at $37^{\circ} \mathrm{C}$ throughout the period of surgery and recovery.

Cerebral blood flow in stroke animals. Cortical blood flow was continuously measured using a laser Doppler flowmeter (PF-5010, Periflux system, SE) in anesthetized animals (Wang et al., 2001b). A Doppler probe $(0.45 \mathrm{~mm}$ in diameter) was stereotaxically placed in the right frontoparietal cortex ( $1.3 \mathrm{~mm}$ posterior, $2.8 \mathrm{~mm}$ lateral to the bregma and 1.0 $\mathrm{mm}$ below the dura).
Behavioral measurements in stroke animals. Animals were placed in an Accuscan activity monitor (Columbus, $\mathrm{OH}$ ) $2 \mathrm{~d}$ after ischemia for behavioral recording (Wang et al., 2001a). The monitor contained 16 horizontal and 8 vertical infrared sensors spaced $2.5 \mathrm{~cm}$ apart. The vertical sensors were situated $10.5 \mathrm{~cm}$ from the floor of the chamber. Motor activities, such as horizontal activity (total number of beam interruptions that occurred in the horizontal sensors), total distance traveled, number of horizontal or vertical movements, movement and rest time, were calculated by the number of beams broken by the animals from 0 to $30 \mathrm{~min}$ after being placed in the chamber.

Triphenyltetrazolium chloride staining in stroke animals. Two days after stroke, some animals were killed for triphenyltetrazolium chloride (TTC) staining (Wang et al., 2001a). The brain tissue was then removed and sliced into $2.0 \mathrm{~mm}$ thick sections. The brain slices were incubated in a $2 \%$ TTC solution. The area of infarction on each brain slice was measured double blind using a digital scanner. The total infarction volume in each animal was obtained from the product of average slice thickness (2 $\mathrm{mm}$ ) and the sum of the area of infarction in all brain slices.

Cytochrome $\mathrm{c}$ in stroke animals. Brain tissues from stroke rats were harvested at $8 \mathrm{hr}$ after the onset of reperfusion for Western blot analysis of cytochrome $c$ (Tsao and Su, 2001). The fourth 2 $\mathrm{mm}$ coronal section $(6-8 \mathrm{~mm}$ from rostra end) from each brain was dissected and homogenized in lysis buffer. We have demonstrated previously that this section contains the largest area of infarction (Chang et al., 2002). The brain-tissue homogenates were centrifuged at $800 \times g$ for $20 \mathrm{~min}$ at $4^{\circ} \mathrm{C}$. The resulting supernatant was additionally centrifuged at $10,000 \times g$ for $20 \mathrm{~min}$ at $4^{\circ} \mathrm{C}$ to obtain the heavy membrane pellet enriched for mitochondria. The supernatants were centrifuged again at $100,000 \times g$ for $60 \mathrm{~min}$ at $4^{\circ} \mathrm{C}$. The resulting supernatant was used as the soluble cytosolic fraction. The mitochondrial pellet was dissolved in lysis buffer and centrifuged at $10,000 \times g$ for $20 \mathrm{~min}$ at $4^{\circ} \mathrm{C}$ to make soluble protein. Fifty micrograms of soluble protein samples from subcellular fractionations were separated by electrophoresis using 10-12\% SDS-PAGE gel. Gels were electrotransferred onto nitrocellulose membranes. The membranes were blocked for $1 \mathrm{hr}$ in TBS/0.05\% Tween 20 containing 5\% nonfat dried milk and then probed with monoclonal mouse anti-cytochrome $c$ antibody (BD PharMingen, San Diego, CA) at a 1:500 dilution at $4^{\circ} \mathrm{C}$ overnight. The amounts of mitochondria were normalized with a mitochondrial marker (voltagedependent anion channel, VDAC). Immunoblot analysis was performed by probing with horseradish peroxidase (HRP)-labeled secondary antibodies, developing with enhanced chemiluminescence (ECL) Western blotting detection reagents, and exposing to Hyperfilm-ECL (Amersham Biosciences, Arlington Heights, IL).

Caspase- 3 enzymatic activity in stroke animals. Activated caspase- 3 enzyme activity was measured using the ApoAlert kit (Clontech) (Wang et al., 2001a). Eight hours after the onset of reperfusion, animals were killed and the brain tissue was removed. The fourth $2 \mathrm{~mm}$ coronal section from each brain was dissected and homogenized in lysis buffer for the same rationale noted previously. Caspase- 3 activity was determined fluorometrically by the formation of 7-amino-4-trifluromethyl coumarin (AFC) from Asp-Glu-Val-Asp-7-amino-4-trifluoromethyl coumarin (DEVD-AFC). The selective caspase-3 inhibitor DEVD-aldehyde (DEVD-CHO) was included, at a concentration of $10 \mu \mathrm{M}$, to ensure that the enzymatic reaction was specific.

6-OHDA lesioning and amphetamine-induced rotation. Rats were anes- 
thetized with chloral hydrate $(0.4 \mathrm{gm} / \mathrm{kg}$, i.p. $)$ and were injected with 6-OHDA $(9 \mu \mathrm{g} / 4 \mu \mathrm{l}$ in normal saline containing $0.2 \mathrm{mg} / \mathrm{ml}$ ascorbic acid) over $4 \mathrm{~min}$, into the left medial forebrain bundle $[-4.4 \mathrm{~mm}$ anteroposterior (AP), 1.2 $\mathrm{mm}$ mediolateral relative to the bregma, and $7.8 \mathrm{~mm}$ below the dura]. One month after lesioning, the unilaterally 6-OHDA-lesioned rats were tested for rotational behavior in response to subcutaneous amphetamine injections (5 $\mathrm{mg} / \mathrm{kg}$ ) in an automated rotometer (Chiang et al., 2001).

TUNEL and tyrosine hydroxylase immunostaining in 6-OHDA-lesioned animals. The 6-OHDA-lesioned animals were killed at $48 \mathrm{hr}$ for TUNEL (Zuch et al., 2000). Some animals treated with 6-OHDA were killed 1 month after 6-OHDA lesioning for tyrosine hydroxylase (TH) immunostaining (Chiang et al., 2001), respectively. The TUNEL assay was conducted according to the manufacturer's recommendations (In Situ Cell Death Detection Kit, Roche). Brains sections (30 $\mu \mathrm{m}$ thick) were immunolabeled using an anti-tyrosine hydroxylase (TH) monoclonal, primary antibody (Boehringer Mannheim, Indianapolis, IN) and either a fluorescent Alexa 568 secondary antibody (Molecular Probes) or an HRP-conjugated secondary antibody (Jackson ImmunoResearch, West Grove, PA). Nuclei were counterstained with 4',6-diamidino-2-phenylindole (Molecular Probes).

In vivo electrochemistry in 6-OHDA-lesioned animals. Six months after lesioning, some 6-OHDA-lesioned animals were anesthetized with urethane $(1.25 \mathrm{gm} / \mathrm{kg}$, i.p.). In vivo chronoamperometric measurements of extracellular dopamine (DA) concentration were performed as described previously (Zhou et al., 1996). The recordings were taken at rates of 10 $\mathrm{Hz}$ continuously using Nafion-coated carbonfiber working electrodes (SF1A: Quanteon, Nicholasville, KY). The release of DA was measured by changes of extracellular DA concentration after the microinjection of $\mathrm{KCl}$ into the striatal parenchyma. $\mathrm{KCl}(70 \mathrm{~mm})$ was applied locally through micropipettes. The working electrode and the micropipette were mounted together with sticky wax; tips were separated by $150 \mu \mathrm{m}$. The electrode/pipette assembly was lowered into striatum (AP $1.0 \mathrm{~mm}$, $2.5 \mathrm{~mm}$ lateral relative to the bregma and $4.0-7.0$ $\mathrm{mm}$ below the dura). Local application of $\mathrm{KCl}$ from the micropipettes was performed by pressure ejection using a pneumatic pump. The ejected volume was monitored by recording the change in the fluid meniscus in the pipette before and after ejection using a dissection microscope.

Blood pressure, heart rate, electrolytes, and blood $\mathrm{CO}_{2}$ measurements. Physiological parameters were measured in 15 rats as described previously (Wang et al., 2001b). Animals were anesthetized with chloral hydrate, and a polyethylene catheter was inserted into the right femoral artery. Mean arterial pressure was recorded through a strain gauge transducer and recorded on a strip chart recorder. Arterial blood $(<1 \mathrm{ml})$ was withdrawn from the artery $30 \mathrm{~min}$ after the intracerebroventricular administration of $\mathrm{AP}_{4} \mathrm{~A}(30 \mathrm{nmol})$ or vehicle. Blood gas, serum electrolytes and glucose were analyzed using standard methods.

Statistical analysis. Statistical analyses were performed with the $t$ test, one- or two-way ANOVA, and the post hoc Newman-Keuls test. Significance was inferred at $p<0.05$. Data are presented as means \pm SEM.

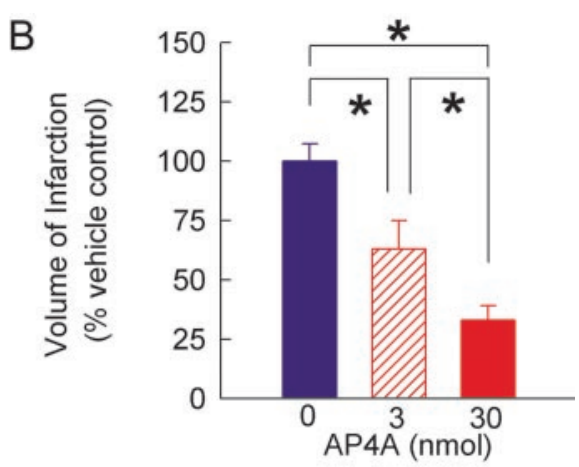

C

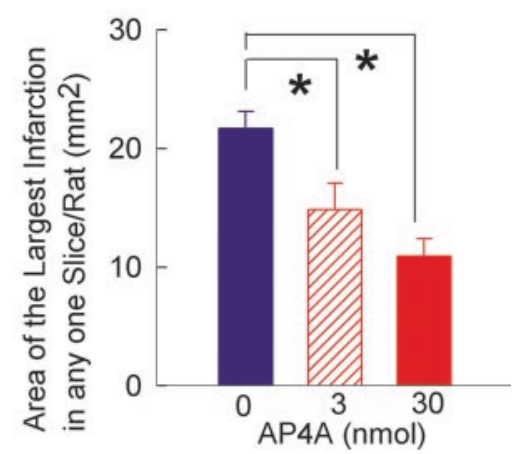

vehicle icv AP4A $30 \mu \mathrm{mol}$ icv

Figure 2. Pretreatment with $\mathrm{AP}_{4} \mathrm{~A}$ reduced ischemia-induced infarction and bradykinesia. $A$, TTC staining indicates marked infarction (white areas) in the right cerebral cortex in a rat pretreated intracerebroventricularly with vehicle. Pretreatment with the largest infarction in a slice in 22 animals $(C)\left({ }^{*} p<0.05\right) . D, A P_{4} A$ significantly increased horizontal activity (hact), total distance traveled (totdist), number of horizontal (movno) or vertical (vmovno) movements, movement time (movtime), while volume of infarction and each locomotor activity from the $\mathrm{AP}_{4} \mathrm{~A}$ group were normalized by comparison with the mean of those indices in stroke animals receiving vehicle.

\section{RESULTS}

\section{Ischemia}

$\mathrm{AP}_{4} A$-induced protection against hypoxia/re-oxygenation in vitro Primary neuronal cultures were treated with vehicle or $\mathrm{AP}_{4} \mathrm{~A}(100$ $\mathrm{nM})$ followed by either hypoxia $\left(3 \% \mathrm{O}_{2}\right.$ for $\left.18 \mathrm{hr}\right) /$ re-oxygenation $\left(21 \% \mathrm{O}_{2}\right.$ for $\left.24 \mathrm{hr}\right)$ or normoxia $\left(21 \% \mathrm{O}_{2}\right.$ for $\left.42 \mathrm{hr}\right) . \mathrm{AP}_{4} \mathrm{~A}$ itself did not alter $\mathrm{LDH}$ production or specific caspase- 3 activity in a normoxic environment ( $p>0.05, t$ test). Nonspecific caspase- 3 activity, defined as the activity found in the presence of the caspase-3 inhibitor DEVD-CHO (inhibitor) in the assay medium, was not detectable. Hypoxia/re-oxygenation enhanced LDH production (Fig. $1 A$ ) and caspase-3 activity (Fig. $1 B$ ) and elicited an increase in TUNEL (Fig. $1 C, D$ ). All of these responses 
A

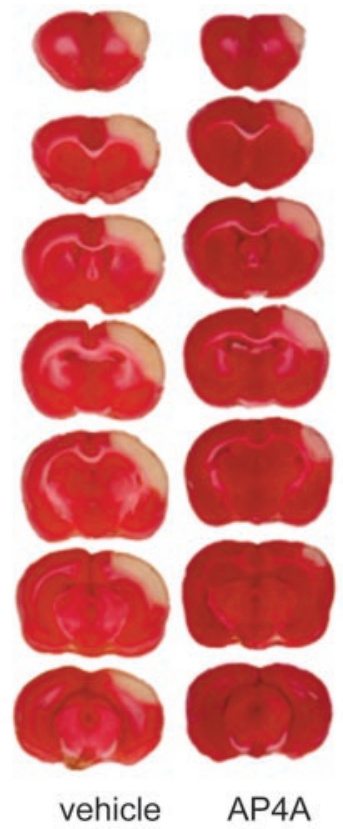

D

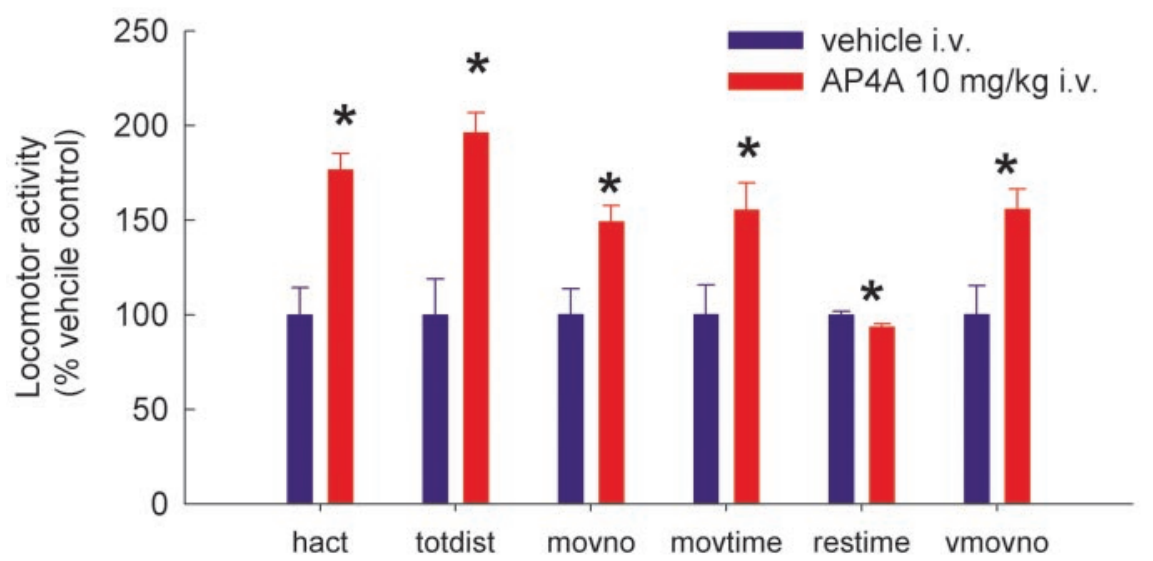

Figure 3. Systemic post-stroke treatment with $\mathrm{AP}_{4} \mathrm{~A}$ reduced ischemia-induced infarction and bradykinesia. $A, \mathrm{TTC}$ staining indicated that the intravenous administration of $A_{4} A(10 \mathrm{mg} / \mathrm{kg}), 5-10 \mathrm{~min}$ after the onset of reperfusion, reduced cerebral infarction. The volume of infarction $(B)$ and the area of the largest infarction $(C)$ in a slice were significantly reduced by intravenous $\mathrm{AP}_{4} \mathrm{~A}$ treatment $(n=17, p<0.05)$. $D$, Intravenous administration of $\mathrm{AP}_{4} \mathrm{~A}$ reduced bradykinesia in stroke animals $\left({ }^{*} p<0.05\right)$.

to hypoxia/re-oxygenation were significantly reduced by pretreatment with $\mathrm{AP}_{4} \mathrm{~A}$ (Fig. $\left.1 A-C\right)(p<0.05$, one-way ANOVA) (Fig. 1D,E).

Pretreatment with $\mathrm{AP}_{4} \mathrm{~A}$ increases locomotor activity and reduces infarction in stroke rats

Rats were pretreated with either vehicle $(n=9)$ or $\mathrm{AP}_{4} \mathrm{~A}(30$ nmol, i.c.v.; $n=10) 20-30$ min before middle cerebral artery occlusion. Animals were individually placed in activity chambers to measure their locomotor activity on the second day after stroke. Pretreatment with $\mathrm{AP}_{4} \mathrm{~A}$, compared with vehicle, significantly enhanced the horizontal activity, total distance traveled, number of horizontal or vertical movements, and movement time in stroke animals $(p<0.05, t$ test) (Fig. $2 D)$.

One hour MCA ligation and $48 \mathrm{hr}$ reperfusion resulted in clear-cut infarction of the cortex in animals pretreated with vehicle or $\mathrm{AP}_{4} \mathrm{~A}$, as examined by TTC staining (Fig. $2 \mathrm{~A}$ ). Infarction was additionally analyzed by two methods: (1) The volume of infarction, which equals $2 \mathrm{~mm}$ [thickness of the slice] $\times$ [sum of the infarction area in all brain slices $\left.\left(\mathrm{mm}^{2}\right)\right]$. (2) The area of the largest infarction in a slice from each rat. In 31 animals studied, pretreatment with $\mathrm{AP}_{4} \mathrm{~A}$ (3 or $30 \mathrm{nmol}$, i.c.v.) dosedependently reduced the volume of infarction (Fig. $2 B)\left(p<0.05, F_{(2,28)}=10.303\right.$, one-way ANOVA and Newman-Keuls test). Similarly, pretreatment with $\mathrm{AP}_{4} \mathrm{~A}$ dose-dependently reduced the area of largest infarction in a given slice (Fig. 2C) $\left(p<0.002, F_{(2,28)}=8.410\right.$, one-way ANOVA; $p<0.05$, post hoc NewmanKeuls test).

Parenteral treatment with $\mathrm{AP}_{4} \mathrm{~A}$ after ischemia

The blood-brain barrier (BBB) can be transiently disrupted during ischemia, which provides a temporal window for the parenteral administration of $\mathrm{AP}_{4} \mathrm{~A}$ to enter the brain. To test the hypothesis that the systemic administration of $\mathrm{AP}_{4} \mathrm{~A}$ may reduce infarct volume, 17 adult rats were subjected to a 60 min right MCA ligation and treated at 5-10 min after removal of the ligature with an intravenous injection of either $\mathrm{AP}_{4} \mathrm{~A}(10 \mathrm{mg} / \mathrm{kg}, n=8)$ or vehicle $(n=9)$. Rats that had been treated with a systemic injection of $\mathrm{AP}_{4} \mathrm{~A}$ had significantly higher locomotor activity than their respective controls (Fig. 3D; $p<0.05, t$ test). Most notably, the volume of infarct, determined at $48 \mathrm{hr}$ after MCA occlusion, was significantly smaller in rats that had been treated with a systemic injection of $\mathrm{AP}_{4} \mathrm{~A}$ early in the reperfusion period (Fig. $3 A-C)(p<0.05, t$ test $)$. These data demonstrate that $\mathrm{AP}_{4} \mathrm{~A}$, given intravenously in the early reperfusion period, exhibits a protective effect against ischemic injury.

Blood gas, electrolytes, and systemic blood pressure

We found that the intracerebroventricular administration of $\mathrm{AP}_{4} \mathrm{~A}(30 \mathrm{nmol} / 20 \mu \mathrm{l})$ did not acutely (25-30 min) alter systemic blood pressure, heart rate, or serum concentrations of glucose, electrolytes, and blood gases ( $p>0.05, t$ test) in 15 non-stroke animals (Fig. $4 A, B$ ).

\section{Cerebral blood flow during stroke}

Cerebral cortical blood flow was measured continuously using a fine $(0.45 \mathrm{~mm}$ diameter) laser Doppler probe. Previous studies have indicated that this modified laser Doppler flowmetry can provide a high degree of spatial and temporal resolution (Wang et al., 2001a,b). We found that cortical blood flow was significantly reduced to the same degree after MCA ligation in both vehicleand $\mathrm{AP}_{4} \mathrm{~A}$-treated animals (Fig. $\left.4 C 1, C 2\right)(p=0.894, t$ test $)$, suggesting that pretreatment of rats with $\mathrm{AP}_{4} \mathrm{~A}$ did not alter cortical blood flow during ischemia.

Translocation of cytochrome $\mathrm{c}$

In vehicle-treated animals $(n=4), 60$ min MCA occlusion and 8 $\mathrm{hr}$ reperfusion resulted in a significant decrease in the levels of 
cytochrome $c$ protein in the mitochondria (Fig. 5A). The intravenous injection of $\mathrm{AP}_{4} \mathrm{~A}(n=3)$ in the early reperfusion period attenuated the reduction in mitochondrial levels of cytochrome $c$ caused by cerebral ischemia and reperfusion $(p<$ $0.05, F_{(3,10)}=357.168$, one-way ANOVA and Newman-Keuls test). The ratio between cytochrome $c$ levels in cytosol and that in mitochondria was significantly increased in the ischemic hemisphere in animals subjected to MCA occlusion and reperfusion, and treated with vehicle. This increase was attenuated by the administration of $\mathrm{AP}_{4} \mathrm{~A}$ in the early reperfusion period. These data suggest that $\mathrm{AP}_{4} \mathrm{~A}$ reduces the ischemia/reperfusioninduced translocation of cytochrome $c$ from the mitochondria into the cytosol, which is thought to be a critical mechanism in apoptosis.

Caspase- 3 activity at $8 \mathrm{hr}$ after ischemia Previous studies have indicated that the activity of caspase-3, a marker of apoptosis, is greatly increased as early as $8 \mathrm{hr}$ after ischemia (Sasaki et al., 2000). To investigate the effect of $\mathrm{AP}_{4} \mathrm{~A}$ on caspase- 3 activity, animals were subjected to $60 \mathrm{~min}$ MCA occlusion and $8 \mathrm{hr}$ of reperfusion, and treated in the early reperfusion period with intravenous $\mathrm{AP}_{4} \mathrm{~A}$ or vehicle. In vehicletreated control animals, MCA-occlusion and reperfusion resulted in a significant increase in the enzymatic activity of caspase-3 (Fig. 5B). Compared with non-stroke animals, MCA occlusion increased caspase- 3 activity $274.1 \pm 34.3 \%$ and $167.5 \pm 25.8 \%$ in the ipsilateral (ischemic side) and contralateral hemisphere, respectively $\left(p<0.05, F_{(2,11)}=43.514\right.$, one-way ANOVA and NewmanKeuls test). Caspase- 3 enzymatic activity was significantly decreased in the brain sections obtained from animals treated with $\mathrm{AP}_{4} \mathrm{~A}$ in the early reperfusion period $\left(p<0.05, F_{(5,24)}=90.736\right.$, one-way ANOVA and Newman-Keuls test) (Fig. 5B).

\section{$P_{1^{-}}, P_{2^{-}}$, and $P_{4^{-} \text {receptor mediation }}$}

Numerous studies have indicated that adenosine has neuroprotective effects against ischemia. We also found that pretreatment with adenosine (30 nmol, i.c.v.) significantly reduced cerebral infarction in eight stroke rats (Fig. 6A) $\left(p<0.05, F_{(2,33)}=\right.$ 17.095, one-way ANOVA and Newman-Keuls test). However, such protective effects of adenosine are much less than those of $\mathrm{AP}_{4} \mathrm{~A}(p<0.05$, one-way ANOVA) (Fig. $6 \mathrm{~A})$, suggesting that mechanisms other than adenosine signaling may be involved. To further characterize the interactions of $\mathrm{AP}_{4} \mathrm{~A}$ with various purinergic $\mathrm{P}_{1^{-}}, \mathrm{P}_{2}$, or $\mathrm{P}_{4}$-receptors, $\mathrm{AP}_{4} \mathrm{~A}$ was co-administered with the selective $\mathrm{P}_{1}$-receptor antagonist sulfonylphenyl theophylline, the nonselective $\mathrm{P}_{2}$-receptor antagonist suramin, or the $\mathrm{P}_{2} / \mathrm{P}_{4}$ receptor antagonist di-inosine pentaphosphate $\left(\mathrm{IP}_{5} \mathrm{I}\right)$. We found that suramin $(n=15)$ did not antagonize the reduction in total infarct volume caused by $\mathrm{AP}_{4} \mathrm{~A}$ (Fig. $\left.6 \mathrm{~B}\right)\left(p>0.05, F_{(2,22)}=\right.$ 2.940, one-way ANOVA). Suramin $(100 \mathrm{nmol})$ induced seizures in six of seven rats and was lethal in three. In contrast to suramin, $\mathrm{AP}_{4} \mathrm{~A}$-induced protection was significantly attenuated by $\mathrm{IP}_{5} \mathrm{I}$ (Fig. $6 C)\left(p<0.05, F_{(2,26)}=6.329\right.$, one-way ANOVA) or sulfo- nylphenyl theophylline (Fig 6D) $\left(p<0.05, F_{(2,23)}=5.103\right.$, oneway ANOVA). These data suggest that activation of $\mathrm{P}_{1^{-}}$and $\mathrm{P}_{4^{-}}$, but not $\mathrm{P}_{2^{-}}$, receptors, is involved in the observed neuroprotective effects of $\mathrm{AP}_{4} \mathrm{~A}$ in the stroke rats.

\section{6-OHDA lesioning}

TUNEL in 6-OHDA-lesioned rats

Six rats were pretreated intracerebroventricularly with $\mathrm{AP}_{4} \mathrm{~A}$ or vehicle and lesioned unilaterally with 6-OHDA in the medial forebrain bundle. Animals were killed $2 \mathrm{~d}$ after 6-OHDA lesioning. An increase in TUNEL was found in the lesioned nigra in rats receiving vehicle pretreatment (Fig. 7A1,A2). TUNEL was mainly colocalized with $\mathrm{TH}$ immunoreactivity in the nigral cells (Fig. 7A3). $\mathrm{AP}_{4} \mathrm{~A}$ pretreatment reduced the density of TUNEL (Fig. 7B1,B2).

\section{Amphetamine-induced ipsilateral rotation in 6-OHDA-}

lesioned rats

Amphetamine-induced rotation was evaluated 1 month after 6-OHDA lesioning in 31 rats. We found that control animals developed a marked ipsilateral rotation after injection (472 \pm 41 turns/hr). This response was significantly attenuated in animals pretreated with $\mathrm{AP}_{4} \mathrm{~A}$ (93 \pm 29 turns/hr, $p<0.05, t$ test).

\section{TH immunostaining in 6-OHDA-lesioned rats}

Immunoreactivity to $\mathrm{TH}$ (Fig. $7 C, D$ ) was used to identify dopaminergic neurons and processes in 12 rats 1 month after lesioning. Very low TH immunoreactivity was noted in the striatum of 
A
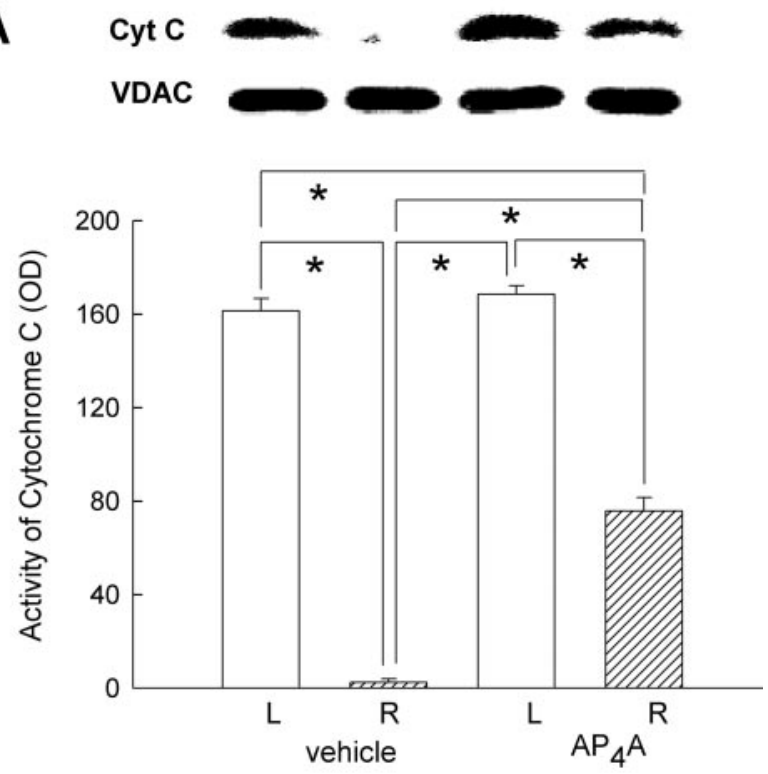

B

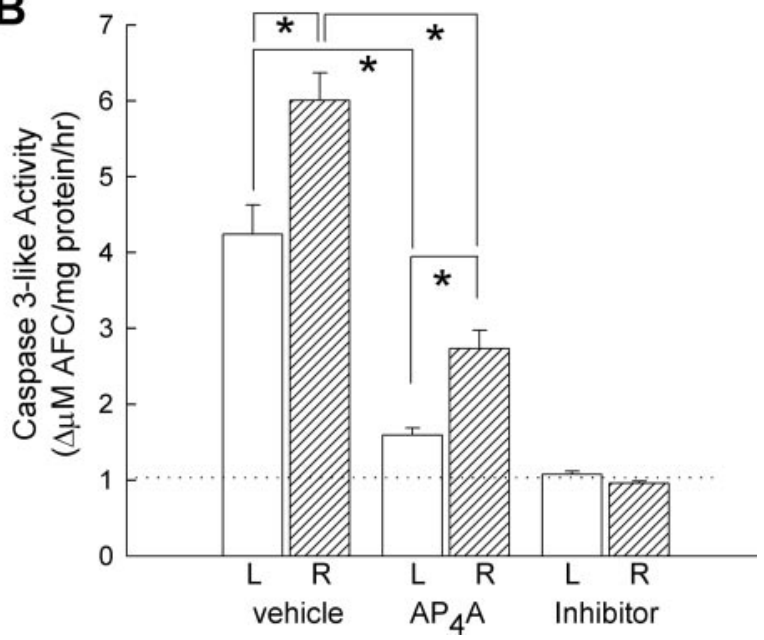

Figure 5. $\quad \mathrm{AP}_{4} \mathrm{~A}$ reduced both translocation of cytochrome $c$ and caspase- 3 activity in stroke brain. $A$, Mitochondrial cytochrome c protein levels were reduced in the ischemic $(R)$ hemisphere of the vehicle-control animals. The intravenous administration of $\mathrm{AP}_{4} \mathrm{~A}$ significantly increased the mitochondrial cytochrome c levels in the ischemic hemisphere compared with vehicle-treated animals. A representative Western blot showing the decrease in mitochondrial cytochrome c (top), but not mitochondrial marker VDAC (bottom), in the lesioned cortex is shown above the histogram. OD, Optical density. B, Increased caspase-3 enzymatic activity was reduced in the ischemic brains of $\mathrm{AP}_{4} \mathrm{~A}$-treated rats at $8 \mathrm{hr}$ after stroke. Caspase-3 enzymatic activity was significantly increased in the ischemic (R) compared with the non-ischemic (L), hemisphere in vehicle-pretreated rats. The administration of $\mathrm{AP}_{4} \mathrm{~A}$ in the early reperfusion period significantly reduced the ischemia-induced increase in caspase- 3 activity. Specific caspase-3 activity, as examined by the addition of the caspase-3 inhibitor DEVD-CHO (inhibitor) in the assay medium, is shown above the dotted line. ${ }^{*} p<0.05$.

6-OHDA-lesioned rats, which had received vehicle (Fig. 7C). There were minimal TH-positive neurons in the lesion-side nigra. In contrast, $\mathrm{AP}_{4} \mathrm{~A}$ partially prevented the loss of $\mathrm{TH}$ immunoreactivity in the 6-OHDA-lesioned striatum and nigra (Fig. 7D).

$\mathrm{KCl}$-induced $\mathrm{DA}$ release in striatum

To determine whether the release of DA was preserved in animals treated with $\mathrm{AP}_{4} \mathrm{~A}, 17$ rats were used to detect dopamine release by in vivo chronoamperometry at 6 months after the injection of 6-OHDA. Of these, 10 rats (four pretreated with $\mathrm{AP}_{4} \mathrm{~A}$ and six with saline) were lesioned with 6-OHDA, whereas seven rats,
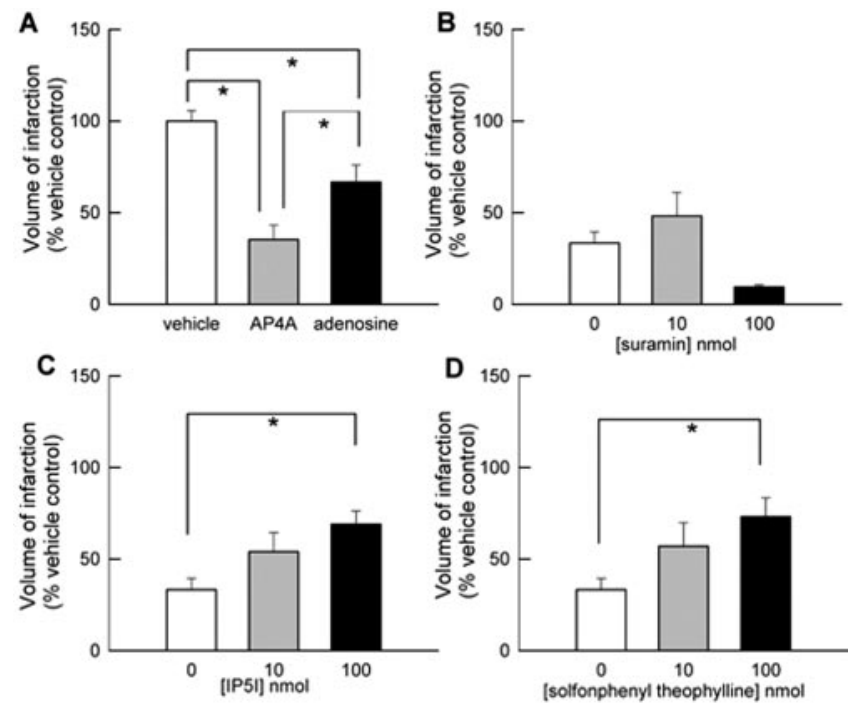

Figure 6. $\mathrm{AP}_{4} \mathrm{~A}$-induced protection against ischemia involves purinergic receptors. $A, A_{4} \mathrm{~A}$ $(30 \mathrm{nmol}$, i.c.v. $)$ is more potent than adenosine $(30 \mathrm{nmol}$, i.c.v.) to reduce cerebral infarction examined at $48 \mathrm{hr}$ after stroke ( $p<0.05$, one-way ANOVA). Co-administration of $\mid \mathbb{P}_{5} I(C)$ or sulfonylphenyl theophylline $(D)$, but not suramin $(B)$, antagonized $\mathrm{AP}_{4} \mathrm{~A}(30 \mathrm{nmol})$-induced protection.

housed in the same environment, served as sham-operated controls. In the sham-operated rats, the local application of $\mathrm{KCl}$ resulted in a significant release of DA in the anterior striatum (Fig. $8 A, B)$. In rats that had been lesioned with $6-\mathrm{OHDA}, \mathrm{KCl}$ caused only a very small release of DA (Fig. $8 A, B$ ). In contrast, the release of DA elicited by $\mathrm{KCl}$ in the striatum of rats treated with $\mathrm{AP}_{4} \mathrm{~A}$ before the administration of 6-OHDA, was significantly greater $(p<0.05$, two-way ANOVA and Newman-Keuls test) (Fig. 8 B). It should be noted that the peak release of DA in 6-OHDAlesioned rats treated with $\mathrm{AP}_{4} \mathrm{~A}$ was significantly reduced compared with the sham-operated rats $(p<0.05$, two-way ANOVA and Newman-Keuls test) (Fig. $8 \mathrm{~B}$ ), suggesting that $\mathrm{AP}_{4} \mathrm{~A}$ attenuates, but does not abolish, the reduction in the release of dopamine caused by 6-OHDA in the striatum.

\section{Discussion}

In this study, we have documented the efficacy of a novel and potent neuroprotective agent $\mathrm{AP}_{4} \mathrm{~A}$ in rodent models of Parkinson's disease and stroke. Pretreatment with $\mathrm{AP}_{4} \mathrm{~A}$ at $100 \mathrm{nM}$, a physiological concentration found in tears (Pintor et al., 2002), reduced hypoxia/reoxygenation-induced increases in $\mathrm{LDH}$ in cortical cultures. Similarly, $\mathrm{AP}_{4} \mathrm{~A}$, given before MCA ligation attenuated cerebral infarction as well as the associated bradykinesia in a dose-related manner in stroke animals. These data suggest that $\mathrm{AP}_{4} \mathrm{~A}$ has protective effects against hypoxic and ischemic injuries in the CNS. Because $\mathrm{AP}_{4} \mathrm{~A}$ did not alter $\mathrm{BP}$, blood gases, and blood electrolytes and did not alter cerebral blood flow during ischemia, the neuroprotective effect of $\mathrm{AP}_{4} \mathrm{~A}$ in vivo is probably not indirectly mediated through the changes in systemic physiological parameters. We found that the application of $\mathrm{AP}_{4} \mathrm{~A}$ even after an ischemic event reduced cerebral infarction and increased motor function. Such a protection may have clinical significance because treatment can be given after the occlusion in stroke patients. Interestingly, animals that received systemic $\mathrm{AP}_{4} \mathrm{~A}$, compared with intracerebroventricular $\mathrm{AP}_{4} \mathrm{~A}$, tended to recover better in one (number of horizontal movements in 30 
$\min , p=0.060, t$ test), but not all, of the locomotor behavioral test parameters. It is possible that the increased number of surgical procedures (or trauma) associated with intracerebroventricular injection may have caused less horizontal movement after stroke.

Pro-apoptotic mechanisms are activated during ischemia-reperfusion. For example, in ischemia or brain trauma, the translocation of cytochrome $c$ from the mitochondria to the cytosol activates caspase- 3 and facilitates apoptosis (Sasaki et al., 2000). Prevention of apoptosis reduces brain damage (Kitagawa et al., 1998; Wang et al., 2001a,b). In the current study, $\mathrm{AP}_{4} \mathrm{~A}$ prevented both translocation of $\mathrm{cy}$ tochrome $c$ and activation of caspase- 3 induced by ischemia/reperfusion. $\mathrm{AP}_{4} \mathrm{~A}$ also reduced hypoxia/reoxygenation-induced TUNEL in cerebrocortical cells. Thus, our data support the view that the neuroprotective effects of $\mathrm{AP}_{4} \mathrm{~A}$ involve the inhibition of several molecular steps that lead to the development of apoptotic cell death during ischemia-reperfusion of the brain.

Previous studies have indicated that $\mathrm{AP}_{4} \mathrm{~A}$ increases intracellular calcium levels through $\mathrm{P}_{2}$ receptors in cortical synaptic terminals and through suramin-insensitive dinucleotide $\mathrm{P}_{4}$ receptors in the midbrain or cerebellum (Pintor et al., 1997). Activation of $\mathrm{P}_{4}$ receptors in rat midbrain synaptic terminals produces an initial voltageindependent calcium entry followed by a voltage-dependent increase in N-type calcium currents (Pintor and Miras-Portugal, 1995). These data suggest that $\mathrm{AP}_{4} \mathrm{~A}$ exhibits differential functions in the cortex and midbrain through the purinergic receptors. In the present study, the neuroprotective effect of $\mathrm{AP}_{4} \mathrm{~A}$ against ischemic injury was reduced by $\mathrm{P}_{1}$ and $\mathrm{P}_{2} / \mathrm{P}_{4}$, but not $\mathrm{P}_{2}$, purinergic antagonists. Thus, it is likely that both $\mathrm{P}_{1^{-}}$and $\mathrm{P}_{4^{-}}$ receptors are involved in $\mathrm{AP}_{4} \mathrm{~A}$-induced protection.

We have demonstrated that the protective effect of $\mathrm{AP}_{4} \mathrm{~A}$ can be reproduced in other models of neuronal injury, in which neurons not located within the cortex are affected. The neurodegeneration induced by 6-OHDA in nigrostriatal dopaminergic neurons was reduced by pretreatment with $\mathrm{AP}_{4} \mathrm{~A}$. All of the effects of 6-OHDA, including motor bias, decrease in TH immunoreactivity in the substantia nigra and striatum, and attenuation of DA release in the striatum, were significantly reduced by pretreatment with $\mathrm{AP}_{4} \mathrm{~A}$. Because 6-OHDA can induce toxicity through the production of reactive oxygen species and apoptosis in nigral neurons (Zuch et al., 2000) and $\mathrm{AP}_{4} \mathrm{~A}$ suppressed the 6-OHDAinduced increase in DNA fragmentation in nigra, we propose that $\mathrm{AP}_{4} \mathrm{~A}$ partially attenuates the toxic effects of 6-OHDA, also through anti-apoptotic mechanism.

In conclusion, our results demonstrate that $\mathrm{AP}_{4} \mathrm{~A}$ has neuroprotective effects against the injuries induced by ischemia in cortex and by 6-OHDA in nigrostriatal dopaminergic neurons. We propose that prevention of the activation of apoptosis by $\mathrm{AP}_{4} \mathrm{~A}$ contributes importantly to the neuroprotective effects of this endogenous compound. These data may well be of clinical importance, insofar as systemic administration of $\mathrm{AP}_{4} \mathrm{~A}$ after an isch-
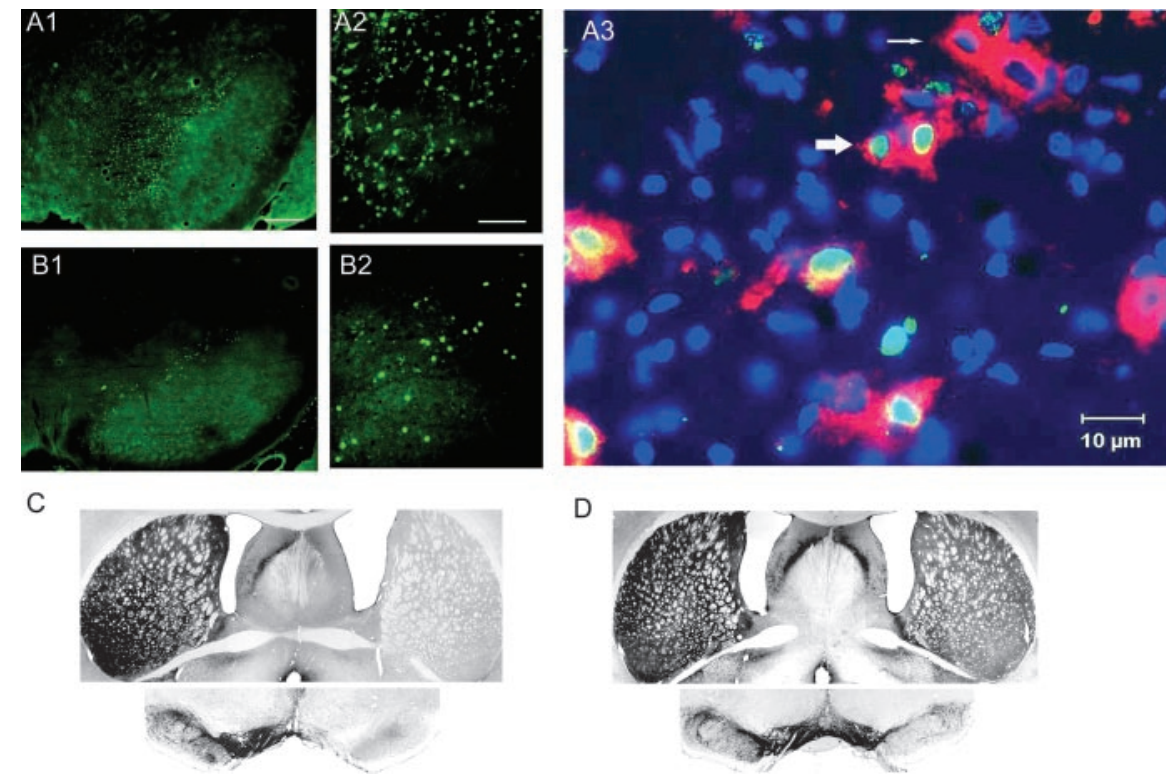
(1)

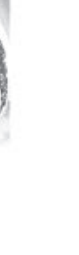

Figure 7. $\mathrm{AP}_{4} \mathrm{~A}$ protects against 6-OHDA-induced lesioning in hemiparkinsonian rats. $A$, Administration of 6-OHDA into the medial forebrain bundle induced TUNEL in the ipsilateral nigral region, $2 \mathrm{~d}$ after the lesioning, in a vehicle-treated animal $(A 1, A 2$, and high magnification). A3, TUNEL (+) labeling (green) was predominantly colocalized to the nuclei (blue) of TH $(+)$ cells 6-0HDA-lesioned animal receiving vehicle pretreatment 1 month after lesioning. $D$, In another 6-0HDA-lesioned animal pretreated with $\mathrm{AP}_{4} \mathrm{~A}$, TH immunoreactivity was relatively preserved in the striatum and nigra.

emic episode may reduce reperfusion injury and improve outcome in patients with stroke.

\section{References}

Ahmet I, Sawa Y, Nishimura M, Ichikawa H, Matsuda H (2000) Diadenosine tetraphosphate (AP4A) mimics cardioprotective effect of ischemic preconditioning in the rat heart: contribution of KATP channel and PKC. Basic Res Cardiol 95:235-242.

Bochner BR, Lee PC, Wilson SW, Cutler CW, Ames BN (1984) AppppA and related adenylylated nucleotides are synthesized as a consequence of oxidation stress. Cell 37:225-232.

Chang CF, Morales M, Chou J, Chen HL, Hoffer BJ, Wang Y (2002) Bone morphogenetic proteins are involved in fetal kidney tissue transplantation -induced neuroprotection in stroke rats. Neuropharmacology 43:418-426.

Chen ST, Hsu CY, Hogan EL, Maricq H, Balentine JD (1986) A model of focal ischemic stroke in the rat: reproducible extensive cortical infarction. Stroke 17:738-743.

Chiang YH, Morales M, Zhou FC, Borlongan CV, Hoffer BJ, Wang Y (2001) Fetal intranigral ventral mesencephalon and kidney tissue bridge transplantation restores the nigrostriatal dopamine pathway in hemiparkinsonian rats. Brain Res 889:200-207.

Emanuelli T, Bonan CD, Sarkis JJ, Battastini AM (1998) Catabolism of Ap4A and Ap5A by rat brain synaptosomes. Braz J Med Biol Res 31:1529-1532.

Khattab M, Hohage H, Hollah P, Rahn KH, Schlatter E (1998) Effects of diadenosine polyphosphates on systemic and regional hemodynamics in anesthetized rats. Kidney Blood Press Res 21:42-49.

Kisselev LL, Justesen J, Wolfson AD, Frolova LY (1998) Diadenosine oligophosphates $(\mathrm{Ap}(\mathrm{n}) \mathrm{A})$, a novel class of signalling molecules? FEBS Lett 427:157-163.

Kitagawa H, Hayashi T, Mitsumoto Y, Koga N, Itoyama Y, Abe K (1998) Reduction of ischemic brain injury by topical application of glial cell line-derived neurotropic factor after permanent middle cerebral artery occlusion in rats. Stroke 29:1417-1422.

Klishin A, Lozovaya N, Pintor J, Miras-Portugal MT, Krishtal O (1994) Pos- 
A
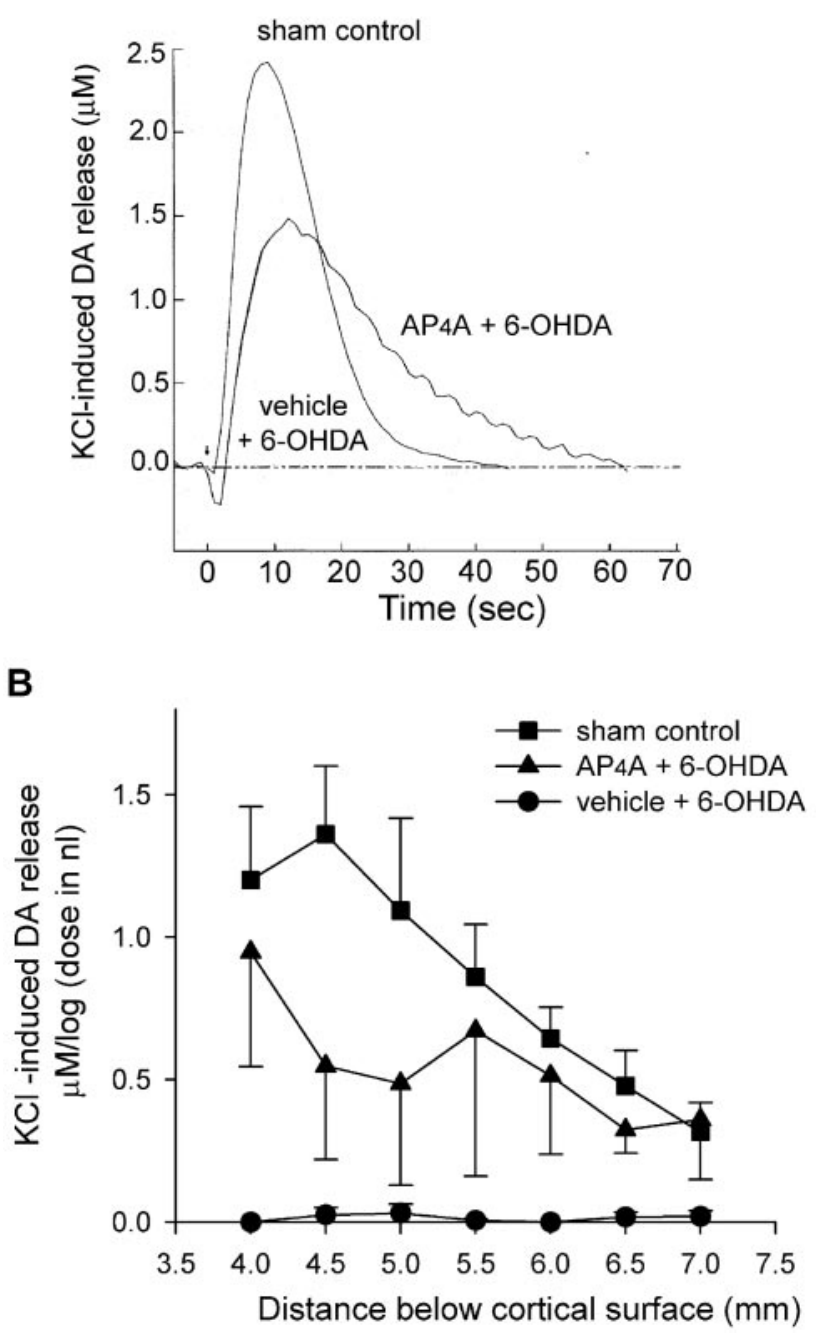

Figure 8. $\quad \mathrm{AP}_{4} \mathrm{~A}$ elevated $\mathrm{KCl}$-induced $\mathrm{DA}$ release in the 6-OHDA-lesioned striatum. $\mathrm{A}$, In vivo chronoamperometric recordings demonstrate that the local application of $70 \mathrm{~mm} \mathrm{KCI}(150 \mathrm{nl})$ to the anterior-dorsal striatum induced DA release in a non-lesioned rat (sham control). Lesioning the medial forebrain bundle abolished $\mathrm{KCl}$-evoked DA release in an animal pretreated with vehicle. DA release was partially preserved by pretreatment with $\mathrm{AP}_{4} A$. Note that $D A$ release in the 6-OHDA-lesioned rat receiving $\mathrm{AP}_{4} A$ pretreatment has a lower amplitude but a longer half-life compared with that in a non-lesioned rat. $B, D$ A release in the anterior striatum (4.0$7.0 \mathrm{~mm}$ below the cortical surface, $2.5 \mathrm{~mm}$ lateral and $1.0 \mathrm{~mm}$ anterior to the bregma) was virtually abolished by $6-0 \mathrm{HDA}$ lesioning in four animals receiving vehicle pretreatment. Pretreatment with $\mathrm{AP}_{4} \mathrm{~A}$ significantly enhanced DA release in 6-0HDA-lesioned rats $(p<0.05$, two-way ANOVA). sible functional role of diadenosine polyphosphates: negative feedback for excitation in hippocampus. Neuroscience 58:235-236.

Lazarowski ER, Watt WC, Stutts MJ, Boucher RC, Harden TK (1995) Pharmacological selectivity of the cloned human P2U-purinoceptor: potent activation by diadenosine tetraphosphate. Br J Pharmacol 116:1619-1627.

Oaknin S, Rodriguez-Ferrer CR, Aguilar JS, Ramos A, Rotllan P (2001) Receptor binding properties of di (1,N6-ethenoadenosine) $5^{\prime}, 5^{\prime \prime \prime}-\mathrm{P} 1, \mathrm{P} 4$ tetraphosphate and its modulatory effect on extracellular glutamate levels in rat striatum. Neurosci Lett 309:177-180.

Pintor J, Miras-Portugal MT (1995) A novel receptor for diadenosine polyphosphates coupled to calcium increase in rat midbrain synaptosomes. Br J Pharmacol 115:895-902.

Pintor J, Diaz-Rey MA, Miras-Portugal MT (1993) Ap4A and ADP-beta-S binding to $\mathrm{P} 2$ purinoceptors present on rat brain synaptic terminals. $\mathrm{Br} \mathrm{J}$ Pharmacol 108:1094-1099.

Pintor J, Porras A, Mora F, Miras-Portugal MT (1995) Dopamine receptor blockade inhibits the amphetamine-induced release of diadenosine polyphosphates, diadenosine tetraphosphate and diadenosine pentaphosphate, from neostriatum of the conscious rat. J Neurochem 64:670-676.

Pintor J, Puche JA, Gualix J, Hoyle CH, Miras-Portugal MT (1997) Diadenosine polyphosphates evoke $\mathrm{Ca} 2+$ transients in guinea-pig brain via receptors distinct from those for ATP. J Physiol 504 (Pt 2):327-335.

Pintor J, Carracedo G, Alonso MC, Bautista A, Peral A (2002) Presence of diadenosine polyphosphates in human tears. Pflugers Arch 443:432-436.

Pivorun EB, Nordone A (1996) Brain synaptosomes display a diadenosine tetraphosphate $(\mathrm{Ap} 4 \mathrm{~A})$-mediated $\mathrm{Ca} 2+$ influx distinct from ATPmediated influx. J Neurosci Res 44:478-489.

Rodriguez-Pascual F, Cortes R, Torres M, Palacios JM, Miras-Portugal MT (1997) Distribution of [3H]diadenosine tetraphosphate binding sites in rat brain. Neuroscience 77:247-255.

Sasaki C, Kitagawa H, Zhang WR, Warita H, Sakai K, Abe K (2000) Temporal profile of cytochrome $c$ and caspase-3 immunoreactivities and TUNEL staining after permanent middle cerebral artery occlusion in rats. Neurol Res 22:223-228.

Tsao LI, Su TP (2001) Hibernation-induction peptide and cell death: [D-Ala2, D-Leu5] enkephalin blocks Bax-related apoptotic processes. Eur J Pharmacol 428:149-151.

Wang Y, Chang CF, Morales M, Chou J, Chen HL, Chiang YH, Lin SZ, Cadet J, Deng X, Wang JY, Chen SY, Kaplan PL, Hoffer BJ (2001a) Bone morphogenetic protein-6 reduces ischemia-induced brain damage in rats. Stroke 32:2170-2178.

Wang Y, Hayashi T, Chang CF, Chiang YH, Tsao LI, Su TP, Borlongan CV, Lin SZ (2001b) Methamphetamine potentiates ischemia/reperfusion insults after transient middle cerebral artery ligation in mice. Stroke 32:775-782.

Zhou FC, Chiang YH, Wang Y (1996) Constructing a new nigrostriatal pathway in the Parkinsonian model with bridged neural transplantation in substantia nigra. J Neurosci 16:6965-6974.

Zuch CL, Nordstroem VK, Briedrick LA, Hoernig GR, Granholm AC, Bickford PC (2000) Time course of degenerative alterations in nigral dopaminergic neurons following a 6-hydroxydopamine lesion. J Comp Neurol 427:440-454. 